\title{
Combining Electronic and Steric Effects To Generate Hindered Propargylic Alcohols in High Enantiomeric Excess
}

\author{
Vijyesh K. Vyas, ${ }^{\dagger, \ddagger}$ Richard C. Knighton, ${ }^{\dagger}$ Bhalchandra M. Bhanage, ${ }^{*}+₫$ and Martin Wills ${ }^{*}{ }^{\dagger}$ \\ ${ }^{\dagger}$ Department of Chemistry, University of Warwick, Coventry CV4 7AL, United Kingdom \\ ${ }^{\ddagger}$ Institute of Chemical Technology, N. Parekh Marg, Matunga, Mumbai 400019, India
}

Supporting Information

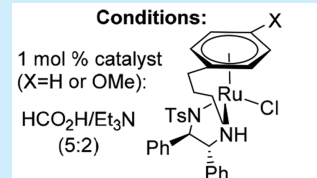

鬲

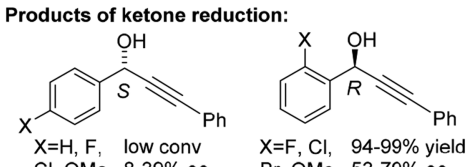

$\mathrm{X}=\mathrm{H}, \mathrm{F}, \quad$ low conv

$\mathrm{X}=\mathrm{F}, \mathrm{Cl}, \quad 94-99 \%$ yield
$\mathrm{Br}, \mathrm{OMe}, 53-79 \%$ ee
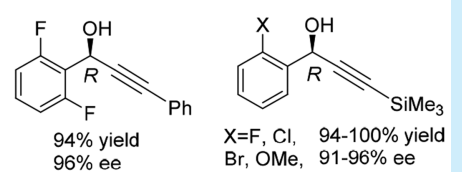

$\begin{array}{ll}\mathrm{X}=\mathrm{F}, \mathrm{Cl}, & 94-100 \% \text { yield } \\ \mathrm{Br}, \mathrm{OMe}, & 91-96 \% \text { ee }\end{array}$

ABSTRACT: Tethered ruthenium-TsDPEN complexes have been applied to the catalysis of the asymmetric transfer hydrogenation of a range of aryl/acetylenic ketones. The introduction of an ortho- substituent to the aryl ring of the substrate results in a reversal of the enantioselectivity, while the introduction of two $o$-fluoro substituents results in an improvement to the reduction enantioselectivity, as does the replacement of a phenyl ring on the alkyne with a trimethylsilyl group. These effects are rationalized as resulting from a change in the steric properties of the aryl ring and the electronic properties of the alkyne which, when matched in the reduction transition state, combine within a "window" of substrate/catalyst matching to generate products of high ee.

A symmetric transfer hydrogenation $(\mathrm{ATH})$ of ketones and imines has been established as a valuable process for the enantioselective synthesis of alcohols and amines. ${ }^{1}$ The breakthrough in 1995 by Noyori et al. with the introduction of arene/ $\mathrm{Ru} / \mathrm{TsDPEN}$ complexes such as $\mathbf{1}^{2}$ led to their subsequent application to numerous synthetic applications ${ }^{3}$ and also to the development of further catalyst derivatives (Figure 1). ${ }^{4-6}$ Of the alternative catalysts developed, the class commonly referred to as "tethered" catalysts such as $2,{ }^{4}$ due to the link between the arene and diamine components, have been extensively adopted in synthetic applications due to their high level of robustness and versatility. The first of these was reported in 2005 by ourselves, ${ }^{4 a}$ with further examples being reported later by Ikariya et al. (DENEB, 3$)^{5}$ and Mohar et al. (N-containing). ${ }^{6}$

The enantioselectivity of ATH using arene/Ru/TsDPEN catalysts, including tethered versions, in reductions of ketones is known to be predominantly influenced by a favorable "edge-face" electronic interaction between electronic-rich groups in the substrate and $\mathrm{H}$ atoms on the electron-deficient $\eta^{6}$-arene ring of the catalyst (Figure 2). ${ }^{7}$ Hence, the reduction of acetophenone derivatives generally takes place in high ee and with a predictable product configuration. The influence of the electronic control alone in ketone ATH is even reflected in the formation of alcohol 6 in $66 \%$ ee. $^{2 a}$ A second class of substrates which are highly compatible with ATH using catalysts $\mathbf{1 - 5}$ are acetylenic ketones, ${ }^{8}$ and with these substrates a similar edge-face interaction is believed to dominate the selectivity (Figure 2).

The enantioselectivity of ketone ATH is also influenced by other factors, notably repulsive effects toward the TS leading to the minor product. ${ }^{7 \mathrm{~d}}$ Recently, it was demonstrated that diarylketones in which one aryl ring contained an orthosubstituent also gave products of high ee in reductions. ${ }^{9}$ This is
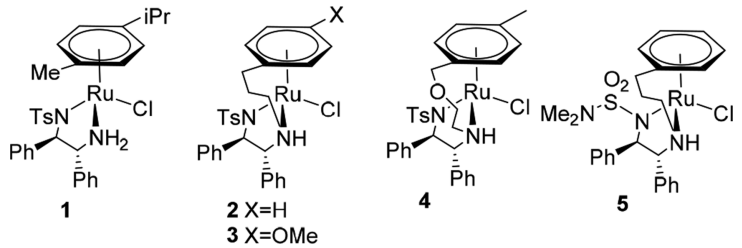

Figure 1. Tethered catalysts for asymmetric reductions of ketones and imines.

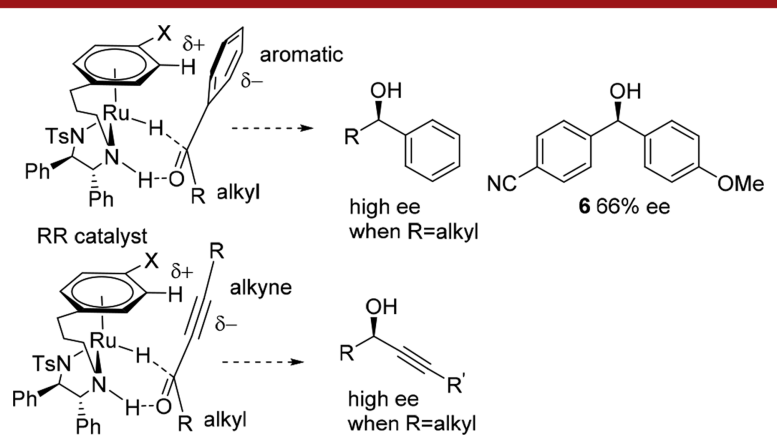

Figure 2. Enantioselectivity control in ATH of acetophenone derivatives and acetylenic ketones.

believed to be the result of the substituted ring adopting an out of plane conformation relative to the ketone, increasing its steric bulk and reducing its conjugation to the ketone. Together, these factors result in the ortho-substituted ring adopting the position

Received: December 13, 2017

Published: January 31, 2018 


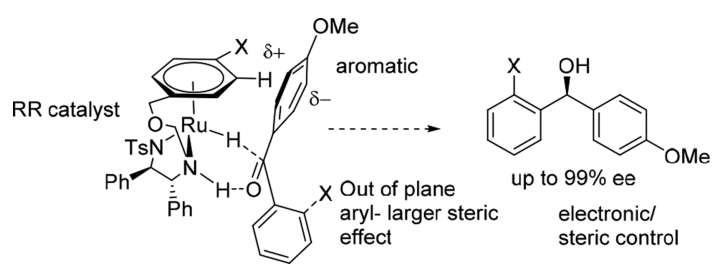

Figure 3. Reduction of biaryl ketones where one aryl group contains an ortho- substituent proceeds in high ee.

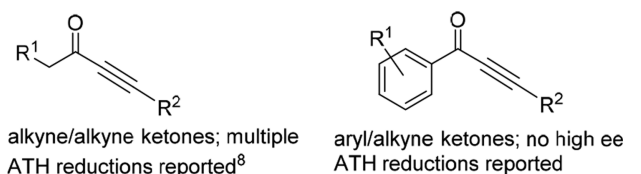

Figure 4. Selective aryl/alkynyl ketone ATH is unreported.

distal to the $\eta^{6}$-arene ring of the catalyst during the reduction (Figure 3). ${ }^{9}$ The same principle has been applied successfully to the reduction of aryl $N$-heteroaryl ketones.

In light of this, we wished to establish whether the same control elements could be transposed to acetylenic ketones. Furthermore, while there are multiple reports of the use of $\mathrm{ATH}^{8}$ (as well as other asymmetric reduction methods ${ }^{11}$ ) for the reduction of alkyl/alkynyl ketones in high ee, at the outset of this study we were aware of no reports of ATH to the reduction of aryl/alkynyl ketones in high ee (Figure 4). Indeed, reports indicate that such substrates give products of low ee when $\mathrm{Ru} / \mathrm{TsDPEN}$ catalysts are used to reduce them. ${ }^{8 \mathrm{c}}$

A literature search revealed few examples of asymmetric reductions of ketones of this type, ${ }^{12-14}$ which have been achieved using the CBS catalyst, ${ }^{12}$ stoichiometric Alpine-Borane and related reagents, ${ }^{13}$ as well as other catalytic methods. ${ }^{14}$

The enantiomerically enriched alcohols formed by reduction of aryl/alkynyl ketones are known to be synthetically valuable and have generally been prepared by the asymmetric catalysis of the addition of alkynes to aldehydes. Extensively, ${ }^{15}$ but not exclusively, ${ }^{16}$ this has been achieved using zinc(II) derivatives of the requisite terminal alkynes. Although this approach does deliver products in high ee's, relatively high levels of catalyst (typically at least $10 \mathrm{~mol} \%$ ) are often required, in addition to $>1$ equiv of a dialkylzinc. Hence, we felt that a solution to the reduction of this obviously challenging class of substrate would create an efficient new route to desirable alcohols and would represent a valuable addition to the field.

Toward this end, we prepared a number of acetylenic ketones for evaluation, and in the first series, we varied only the parasubstituent on the aromatic substituent (Scheme 1, Figure 5). These were reduced, using catalysts 2 and 3, in low conversion and ee; the result of both the aryl ring and the triple bond

Scheme 1. Asymmetric Reductions of Aryl/Acetylenic Ketones in This Study

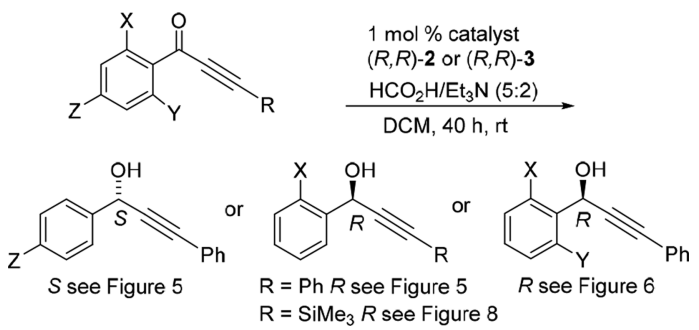

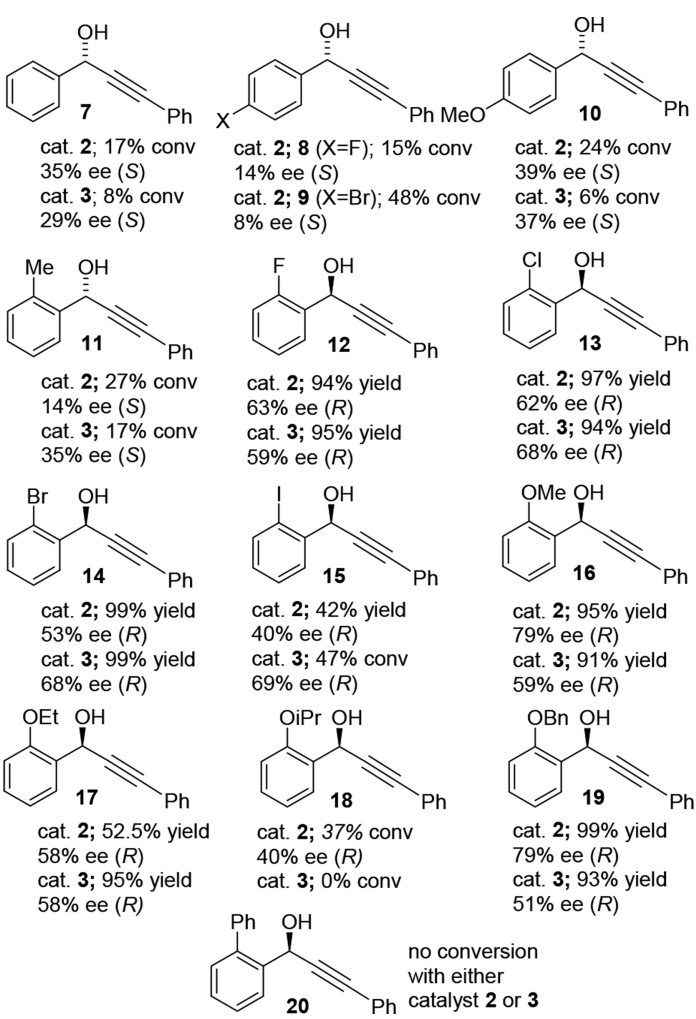

Figure 5. Products of ATH of para- and ortho-substituted aryl/acetylenic ketones.

"competing" for the "edge-face" position in the catalyst (Figure 2 ). The absolute configurations in the case of the known products 7-10 were readily established through the use of reported HPLC resolution protocols as $S$, which correlated to a favored interaction of the aryl ring in the substrate with the $\eta^{6}$-arene of the catalyst (Figure 2). The details of literature correlations for these and other products, where known, are given in the Supporting Information.

We next introduced an ortho-substituent to the aryl ring of the substrate (Figure 5). The ee's of reduction products 11-19 were moderate to good (up to ca. $79 \%$ ee) but, more significantly, in the case of all except product $\mathbf{1 1}$ the configuration was shown to be $R$, i.e., reversed relative to 7 . This indicates that the triple bond in these substrates now favors the position adjacent to the $\eta^{6}$-arene in the transition state (Figure 2), possibly for reasons analogous to the diaryl ketone featured in Figure 3.

At this stage, we also compared a number of ATH catalysts in the reduction to give alcohol 16 as a representative example (SI). The ether-linked catalyst DENEB 4, a Ms-substituted 3C tethered catalyst, and a C4-tethered catalyst gave less satisfactory results. Raising the temperature also reduced the enantioselectivity of the reduction. As a result of this study, we elected to continue our studies with the two $3 \mathrm{C}$ catalysts ( 2 and 3 ) used in the tests highlighted above.

Encouraged by the observations, we added two orthosubstituents to our substrate (Figure 6). While the 2,6-difluorosubstituted substrate gave product $\mathbf{2 1}$ cleanly in high yield and ee, the major product from reduction of the 2,6-dichloro analogue was the saturated ketone 1-(2,6-dichlorophenyl)-3-phenylpropanone, with the alcohol 22 formed in just $8-10 \%$ conversion (but high ee). A limitation for any reduction to occur, however, is the requirement for electron-withdrawing groups in the substrate; electron-rich substrates were reduced in low conversion if at all 


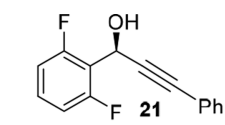

cat. 2; $94 \%$ yield, $94 \%$ ee $(R)$ cat. $3 ; 94 \%$ yield, $94 \%$ ee $(R)$

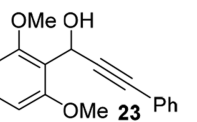

cat 2; $8 \%$ conv, $20 \%$ ee

cat. 3; $0 \%$ conv

Figure 6. Products of ATH of di-ortho-substituted aryl/acetylenic ketones. The configuration of $\mathbf{2 3}$ and $\mathbf{2 4}$ was not established.
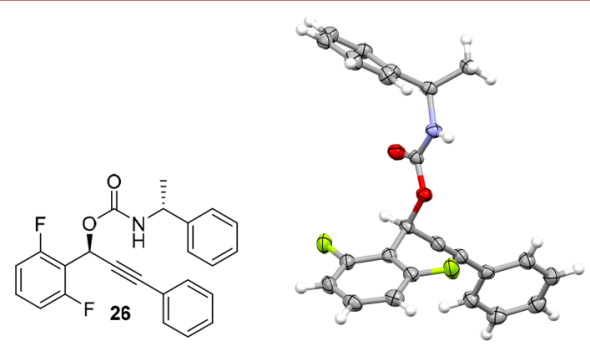

Figure 7. X-ray crystallographic stucture of $\mathbf{2 6 .}$

and in generally poor ee accompanied by inseparable side products, which were not characterized. The results reflect the extra steric hindrance in these substrates.

Since there are very few reports on the asymmetric synthesis of the alcohols shown in Figure 6, an X-ray crystallographic structure of a diastereoisomeric derivative of $\mathbf{2 1}$ was obtained (Figure 7) which confirmed its absolute configuration as $R$. The dichloride analogue 22 was assigned by analogy; however, the low conversions and ee's made the assignment of the electron-rich derivatives difficult to assign with certainty.

To see if the selectivity could be increased further, we increased the electron density at the alkyne by replacing the phenyl ring at its terminus. Although the use of an $\mathrm{nBu}$ group gave no improvement (27), use of an electron-releasing trimethylsilyl group, however, increased the ee of the products significantly in some cases in up to 96\% ee (Figure 8 ).

Furthermore, both electron-withdrawing and electron-donating groups on the aromatic ring were tolerated, although in

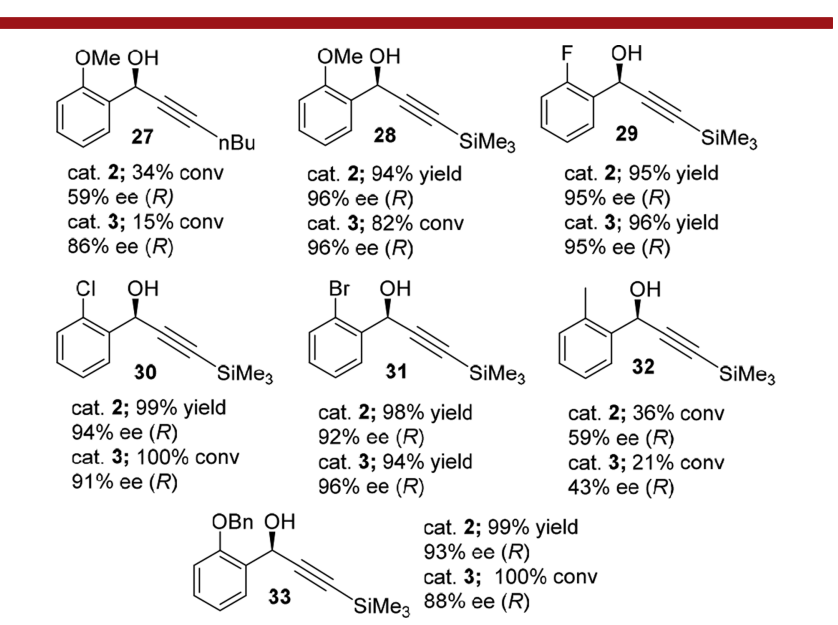

Figure 8. Products of ATH of ortho-substituted aryl/(trimethylsilyl)acetylenic ketones.

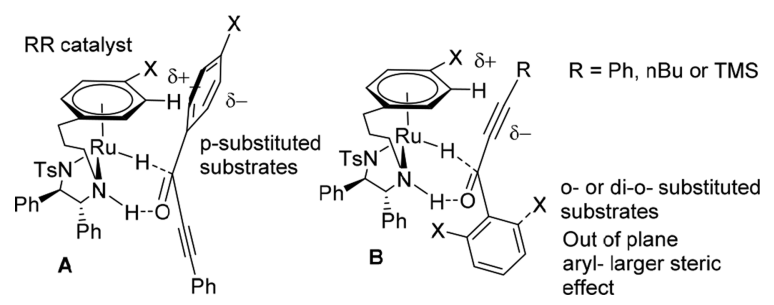

Figure 9. Proposed favored orientations of transition states of substrates containing para- (A) and ortho-substituents (B) on the aryl ring during ATH. ${ }^{9,10}$

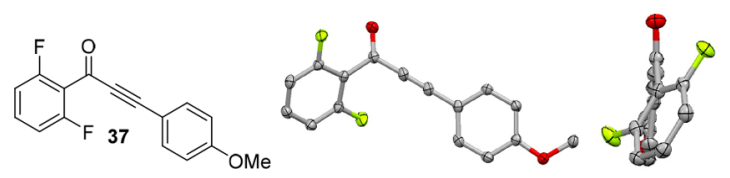

Figure 10. Structure of ketone 37.

Scheme 2. Formal Synthesis of an Allocolchicine Analogue

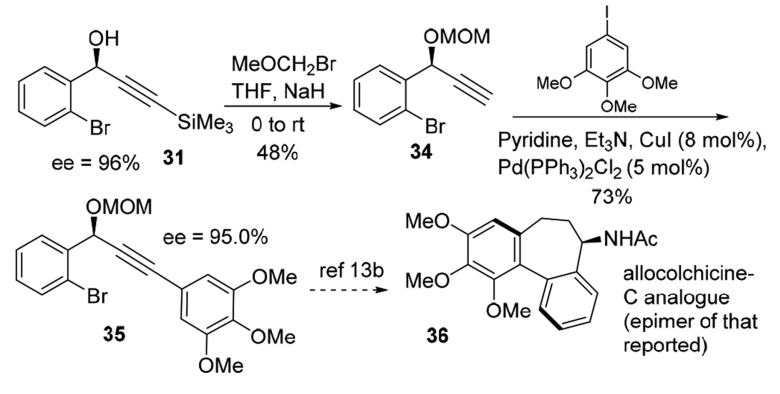

contrast a simple ortho-methyl-containing substrate gave a product (32) in low conversion and ee.

Figure 9 contains a summary of the likely orientation of substrate relative to the catalysts for reductions to the products illustrated in Figures 5, 6, and 8. In the case of para-substituted substrates, the aromatic ring predominately occupies the position adjacent to the $\eta^{6}$-arene ring (Figure 9A). However, when the aromatic ring of the substrate is ortho-substituted it will be twisted relative to the plane of the ketone, in analogy with the examples in Figure 3. ${ }^{9,10}$ In these situations, the alkyne occupies the position adjacent to the $\eta^{6}$-arene ring instead (Figure 9B). This preference is further reinforced by the introduction of the electron-releasing TMS group at the end of the triple bond, hence the higher ee's observed for the products in Figure 8.

In an attempt to gain evidence of the postulated reduction of the planarity of the ortho-substituted arenes with the carbonyl substituent, we prepared ketone 37 and studied it by X-ray diffraction (Figure 10). The solid-state structure of 37 shows significant deviation from planarity with dihedral angles of $41.9^{\circ}$ and $-37.8^{\circ}$ for the crystallographically distinct molecules. This out-of-plane twist, which is corroborated by existing structures in the literature, ${ }^{17}$ supports the selectivity observed in this class of compound, driven by dominant alkyne-catalyst interactions.

To demonstrate the value of the products of the highly selective ATH, alcohol 31 was readily converted via 34 (MOM protection was accompanied by desilylation) to the key intermediate 35 used in a total synthesis of allocolchicine analogue 36 (Scheme 2). ${ }^{13 \mathrm{~b}}$ Since the precursor to 31 can be prepared in one step from $o$ bromobenzoyl chloride, ${ }^{18}$ the route to 35 takes just four steps in total. The route also replaces a stoichiometric process, which requires large quantities of reagent relative to substrate, with one 
requiring just $1 \mathrm{~mol} \%$ of an ATH catalyst. Completion of the formal total synthesis also provided a means to confirm the absolute stereochemistry of $\mathbf{3 1}$ as $R$, through comparison of the sign of optical rotation, and the HPLC data, to that previously reported. $^{13 \mathrm{~b}}$

In conclusion, we have demonstrated that ATH can be used effectively to reduce aryl/alkynyl ketones in high enantiomeric excess and conversion, provided that the substrate satisfies certain requirements which place them in a "window" of high compatibility with the catalyst. The results demonstrate that the low initial enantioselectivity on the simple unsubstituted compound 7 can be improved and optimized through understanding of the factors governing stereocontrol.

\section{ASSOCIATED CONTENT}

\section{(S) Supporting Information}

The Supporting Information is available free of charge on the ACS Publications website at DOI: 10.1021/acs.orglett.7b03884.

Experimental procedures, NMR spectra, and HPLC and Xray data $(\mathrm{PDF})$

\section{Accession Codes}

CCDC 1574558 and 1582072 contain the supplementary crystallographic data for this paper. These data can be obtained free of charge via www.ccdc.cam.ac.uk/data_request/cif, or by emailingdata_request@ccdc.cam.ac.uk, or by contacting The Cambridge Crystallographic Data Centre, 12 Union Road, Cambridge CB2 1EZ, UK; fax: +44 1223336033.

\section{AUTHOR INFORMATION}

\section{Corresponding Authors}

*E-mail: m.wills@warwick.ac.uk.

*E-mail: bm.bhanage@ictmumbai.edu.in.

ORCID $\odot$

Bhalchandra M. Bhanage: 0000-0001-9538-3339

Martin Wills: 0000-0002-1646-2379

Notes

The authors declare no competing financial interest.

\section{ACKNOWLEDGMENTS}

V.K.V. acknowledges the PhD funding agency Department of Science \& Technology (DST) INSPIRE (SRF) as well as the Newton Bhabha fund of the Royal Society and Warwick University. EPSRC is thanked for a project grant (EP/ M006670/1) (R.C.K.). We thank Johnson Matthey Catalysts for a gift of catalyst $\mathbf{2}$. Crystallographic data for $\mathbf{2 6}$ were collected using instruments purchased through support from Advantage West Midlands and the European Regional Development Fund. Crystallographic data for 37 were collected using an instrument funded by the ERC under the European Union's Horizon 2020 research and innovation programme (Grant No. 637313).

\section{REFERENCES}

(1) (a) Wang, D.; Astruc, D. Chem. Rev. 2015, 115, 6621. (b) Milner, L.; Talavera, G.; Nedden, H. Chem. Oggi 2017, 35, 37.

(2) (a) Noyori, R.; Hashiguchi, S. Acc. Chem. Res. 1997, 30, 97. (b) Fujii, A.; Hashiguchi, S.; Uematsu, N.; Ikariya, T.; Noyori, R. J. Am. Chem. Soc. 1996, 118, 2521.

(3) (a) Duan, S.; Li, B.; Dugger, R. W.; Conway, B.; Kumar, R.; Martinez, C.; Makowski, T.; Pearson, R.; Olivier, M.; Colon-Cruz, R. Org. Process Res. Dev. 2017, 21, 1340. (b) Wu, L.; Jin, R.; Li, L.; Hu, X.; Cheng,
T.; Liu, G. Org. Lett. 2017, 19, 3047. (c) Zheng, D.; Zhao, Q.; Hu, X.; Cheng, T.; Liu, G.; Wang, W. Chem. Commun. 2017, 53, 6113. (d) Fang, L.; Liu, S.; Han, L.; Li, H.; Zhao, F. Organometallics 2017, 36, 1217. (e) Hong, Y.; Chen, J.; Zhang, Z.; Liu, Y.; Zhang, W. Org. Lett. 2016, 18, 2640. (f) Vyas, V. K.; Bhanage, B. M. Org. Chem. Front. 2016, 3, 614.

(4) (a) Nedden, H. G.; Zanotti-Gerosa, A.; Wills, M. Chem. Rec. 2016, 16, 2623. (b) Forshaw, S.; Matthews, A. J.; Brown, T. J.; Diorazio, L. J.; Williams, L.; Wills, M. Org. Lett. 2017, 19, 2789. (c) Fang, Z.; Wills, M. Org. Lett. 2014, 16, 374. (d) Vyas, V. K.; Bhanage, B. M. Org. Lett. 2016, $18,6436$.

(5) (a) Touge, T.; Hakamata, T.; Nara, H.; Kobayashi, T.; Sayo, N.; Saito, T.; Kayaki, Y.; Ikariya, T. J. Am. Chem. Soc. 2011, 133, 14960. (b) Parekh, V.; Ramsden, J. A.; Wills, M. Catal. Sci. Technol. 2012, 2, 406.

(6) Kišić, A.; Stephan, M.; Mohar, B. Org. Lett. 2013, 15, 1614. (b) Kišić, A.; Stephan, M.; Mohar, B. Adv. Synth. Catal. 2014, 356, 3193.

(7) (a) Yamakawa, M.; Yamada, I.; Noyori, R. Angew. Chem., Int. Ed. 2001, 40, 2818. (b) Casey, C. P.; Johnson, J. B. J. Org. Chem. 2003, 68, 1998. (c) Brandt, P.; Roth, P.; Andersson, P. G. J. Org. Chem. 2004, 69, 4885. (d) Dub, P. A.; Gordon, J. C. Dalton Trans. 2016, 45, 6756.

(8) (a) Matsumura, K.; Hashiguchi, S.; Ikariya, T.; Noyori, R. J. Am. Chem. Soc. 1997, 119, 8738. (b) Takahashi, K.; Arai, Y.; Honda, T. Tetrahedron Lett. 2017, 58, 4048. (c) Arai, N.; Satoh, H.; Utsumi, N.; Murata, K.; Tsutsumi, K.; Ohkuma, T. Org. Lett. 2013, 15, 3030. (d) Siva Nagi Reddy, K.; Sabitha, G. Tetrahedron Lett. 2017, 58, 1198. (e) Kumaraswamy, G.; Narayanarao, V.; Shanigaram, P.; Balakishan, G. Tetrahedron 2015, 71, 8960. (f) Brandt, D.; Dittoo, A.; Bellosta, V.; Cossy, J. Org. Lett. 2015, 17, 816. (g) Fang, Z.; Wills, M. J. Org. Chem. 2013, 78, 8594.

(9) Touge, T.; Nara, H.; Fujiwhara, M.; Kayaki, Y.; Ikariya, T. J. Am. Chem. Soc. 2016, 138, 10084.

(10) Wang, B.; Zhou, H.; Lu, G.; Liu, Q.; Jiang, X. Org. Lett. 2017, 19, 2094.

(11) Ramachandran, P. V.; Teodorovic, A. V.; Rangaishenvi, M. V.; Brown, H. C. J. Org. Chem. 1992, 57, 2379.

(12) (a) Naveen, N.; Koppolu, S. R; Balamurugan, R. Adv. Synth. Catal. 2015, 357, 1463. (b) Yu, C.-M.; Kim, C.; Kweon, J.-H. Chem. Commun. 2004, 2494. (c) Tian, T.; Li, J.; Xue, J.; Zhang, J.; Li, Y. J. Org. Chem. 2015 $80,4189$.

(13) (a) Georgy, M.; Boucard, V.; Campagne, J.-M. J. Am. Chem. Soc. 2005, 127, 14180. (b) Leblanc, M.; Fagnou, K. Org. Lett. 2005, 7, 2849. (c) Grillet, F.; Brummond, K. M. J. Org. Chem. 2013, 78, 3737.

(14) (a) Hamzic, M.; Pietruszka, J.; Sandkuhl, D. Chirality 2011, 23, E110. (b) Eagon, S.; DeLieto, C.; McDonald, W. J.; Haddenham, D.; Saavedra, J.; Kim, J.; Singaram, B. J. Org. Chem. 2010, 75, 7717.

(15) (a) Sasaki, H.; Boyall, D.; Carreira, E. M. Helv. Chim. Acta 2001, 84, 964. (b) Frantz, D. E.; Fässler, R.; Carreira, E. M. J. Am. Chem. Soc. 2000, 122, 1806. (c) Anand, N. K.; Carreira, E. M. J. Am. Chem. Soc. 2001, 123, 9687. (d) El-Sayed, E.; Anand, N. K.; Carreira, E. M. Org. Lett. 2001, 3, 3017. (e) Huang, W.-C.; Liu, W.; Wu, X.-D.; Ying, J.; Pu, L. J. Org. Chem. 2015, 80, 11480. (f) Song, T.; Zheng, L.-S.; Ye, F.; Deng, W.-H.; Wei, Y.L.; Jiang, K.-Z.; Xu, L.-W. Adv. Synth. Catal. 2014, 356, 1708. (g) Zheng, B.; Li, Z.; Liu, F.; Wu, Y.; Shen, J.; Bian, Q.; Hou, S.; Wang, M. Molecules 2013, 18, 15422.

(16) (a) Ito, J.-I.; Asai, R; Nishiyama, H. Org. Lett. 2010, 12, 3860. (b) Zhang, A.-L.; Yang, L.-W.; Yang, N.-F.; Liu, D.-C. J. Organomet. Chem. 2014, 768, 50. (c) Takita, R.; Yakura, K.; Ohshima, T.; Shibasaki, M. J. Am. Chem. Soc. 2005, 127, 13760. (d) Corey, E. J.; Cimprich, K. A. J. Am. Chem. Soc. 1994, 116, 3151.

(17) (a) Braun, R. U.; Ansorge, M.; Müller, T. J. J. Chem. - Eur. J. 2006, 12, 9081. (b) Prabuswamy, M.; Kumar, S. M.; Bhuvaneshwar, D.; Murthy, C. S. S. S.; Lokanath, N. K. Acta Crystallogr., Sect. E: Struct. Rep. Online 2012, 68, o3190. (c) Schwarzer, A.; Seichter, W.; Weber, E.; Stoeckli-Evans, H.; Losada, M.; Hulliger, J. CrystEngComm 2004, 6, 567.

(18) (a) Karpov, A. S.; Mueller, T. J. J. Org. Lett. 2003, 5, 3451. (b) Carmichael, R. A.; Sophanpanichkul, P.; Chalifoux, W. A. Org. Lett. 2017, 19, 2592. 